\title{
Notching up vascular regeneration
}

\author{
Cell Research (2014) 24:777-778. doi:10.1038/cr.2014.68; published online 23 May 2014
}

\begin{abstract}
Regenerative medicine for cardiovascular disease requires effective approaches for therapeutic revascularization. In a recent paper in Cell Research, Sahara et al. establish a new role for Notch signaling to promote endothelial progenitor differentiation, and develop a protocol based on Notch inhibition in endothelial progenitors to markedly enhance the yield and purity of functional endothelial cells differentiated from embryonic stem cells.
\end{abstract}

Cardiovascular disease is the world's leading cause of death and a major socioeconomic burden. Many cardiovascular ailments, such as ischemic heart disease, stroke, and peripheral vascular disease, are caused by loss of vascular supply, which causes irreversible cell loss and organ failure. Successful treatment of these diseases through traditional or regenerative approaches will require re-establishing normal vascular beds, both for proper nutrient exchange and for essential paracrine signals exchanged between vascular cells and organ stroma. However, vascular regeneration has been a difficult challenge, in part because of inadequate means to efficiently and rapidly generate endothelial cells (ECs) for replacement therapy or for in vitro proangiogenic drug screening. Human pluripotent stem cells (PSCs) are an attractive source of ECs, because the cells can be propagated and expanded indefinitely in culture. Furthermore, the advent of induced pluripotent stem cells (iPSCs) raises the possibility of generating autologous ECs. Several studies have reported differentiation of PSCs into ECs, but efficiencies and yields have been low $(<3 \%)$ [1-3]. In a recent paper published in Cell Research, Sahara, Chien and colleagues overcome this barrier by reporting a protocol to efficiently and robustly generate stem cell-derived endothelial progenitors (EPs) and functional ECs [4].

The advance of Sahara et al. [4] was made by adopting a staged differentiation strategy analogous to that used for efficient differentiation of human embryonic stem cells (hESC) into cardiomyocytes, islet cells, and other terminally differentiated cell types. Sahara et al. conceptualized hESC-toEC differentiation as occurring in three phases (Figure 1), and systematically investigated conditions and signaling pathway modulators to optimize the first two phases. In phase 1, PSCs differentiate into mesodermal precursors, which commit to EPs in phase 2, and then differentiate into mature endothelial cells (ECs) in phase 3. The EPs formed and amplified in phase 2 are critical for efficient generation of ECs from PSCs. The authors characterized this population and showed that the subset with the greatest vasculogenic activity had the surface marker phenotype $\mathrm{CHD}^{+} \mathrm{CD} 31^{+} \mathrm{KDR}^{+} \mathrm{CD} 34^{+} \mathrm{CD} 14^{-}$. These EPs were highly proliferative, clonogenic, formed functional ECs, and expressed genes characteristics of EPs, including NRP1, an established marker of proliferative and long surviving EPs [5]. Identification of this surface marker phenotype of the key EP stage will facilitate further studies to enhance their generation, amplification, differentiation, and deployment for therapeutic vascularization.

Building on this intellectual framework, the authors sought to optimize generation and amplification of EPs in phases 1 and 2. Previous work established that BMP4 in phase I and VEGF-A in phase 2 promotes hESC differentiation into ECs, albeit with low efficiency ( $1 \%-3 \%$ ECs on day 6-7) [1-3]. By screening over 60 bioactive compounds, the authors found compounds that synergistically enhanced EC differentiation. In phase 1, addition of glycogen synthase kinase-3 $\beta$ inhibitors $\mathrm{BIO}$ or $\mathrm{CP} 21 \mathrm{R} 7$ potentiated Wnt signaling and enhanced induction of mesodermal progenitors. In phase 2 , EP commitment and expansion were enhanced by DAPT, a small-molecule inhibitor of $\gamma$-secretase, which is required for Notch signaling. DAPT or shRNA knockdown of Notch signaling pathway components synergized with VEGF-A to stimulate EP expansion, so that by the end of day 6 EPs constituted $50 \%$ of all cells. Addition of placental growth factor (PLGF) and hepatocyte growth factor (HGF) in phase 2 further enhanced EC yield. Subsequent removal of Notch inhibitor in phase 3 permitted differentiation of the expanded EPs into large numbers of functional ECs.

The authors further investigated the mechanism by which Notch inhibition amplifies EPs. The Notch signaling pathway is a well-established regulator of mature ECs [6]. Inactivation of Notch ligands or receptors causes lethal vascular defects in developing embryos. The interplay of Notch and VEGF-A signaling is known to be crucial for angiogenic sprouting of mature ECs: VEGF-A signaling through KDR stimulates sprouting tip ECs to express DLL4, which activates Notch in neighboring stalk cells to dampen their VEGF-A responsiveness [6]. The authors uncovered a new role for 


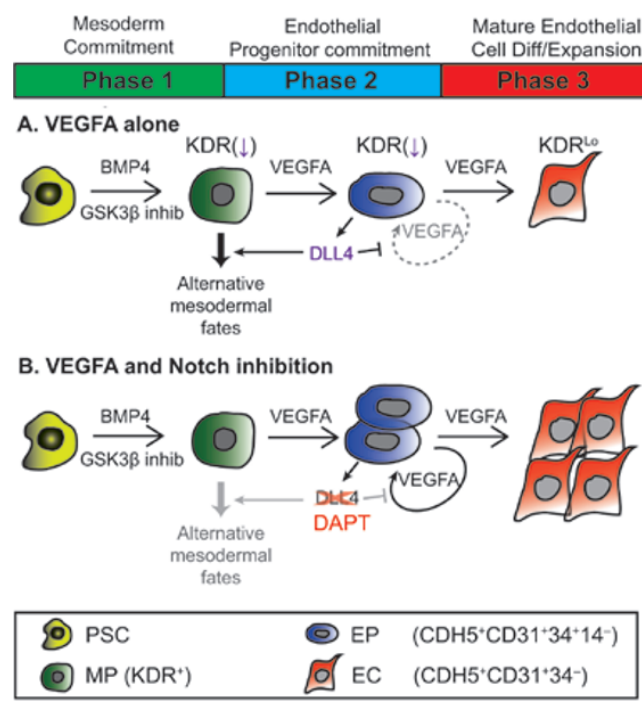

Figure 1 Notch inhibition augments endothelial progenitor amplification in a three-phase pluripotent stem cell differentiation protocol. Pluripotent stem cells (PSCs) differentiate to endothelial cells (ECs) through a three-phase protocol. Phase 1 produces mesodermal progenitors (MP), which are then committed to endothelial progenitors (EPs) and amplified in phase 2. In phase 3, endothelial progenitors differentiate into ECs. VEGFA actviates the Notch signaling pathway in phase 2, inhibiting expression of KDR (the VEGF receptor; purple down arrows), redirecting cells to alternative fates and preventing EP amplification (A). Notch inhibition (DAPT) sustained KDR expression, favoring generation and amplification of EPs to enhance final EC yield (B).

crosstalk of VEGF and Notch signaling pathways in EPs. They showed that VEGF-A signaling through KDR in EPs activates Notch, which feeds back to inhibit KDR transcription and thereby limits VEGF-KDR-dependent EP maintenance and amplification. The authors found that Notch also limits mesodermal progenitor differentiation to EPs and favors their differentiation into alternative lineages. As a result of these two mechanisms, VEGF-A stimulation without Notch inhibition in phase 2 results in low EP and EC yield (Figure 1A). In contrast, VEGFA stimulation with Notch inhibition enhances EP formation, maintenance and amplification, ultimately boosting EC yield (Figure 1B).

The authors assessed the function of stem cell-derived EPs in two in vivo angiogenesis models. In the matrigel plug model, transplanted EPs formed neo-vessels that functionally anastomosed to host vessels and continued to function for over 3 months. Importantly, transplanted EPs had a 3-5-fold greater capacity to form vascular networks in vivo after grafting compared to either stem cell-derived or human umbilical vein mature ECs. In a myocardial infarction model, delivery of EPs at the time of experimental coronary artery ligation attenuated LV remodel- ing and increased capillary density, both through direct formation of ECs incorporated into host vessels and through enhanced native vessel EC density. These data demonstrate that the PSC-derived EPs are functional, and that transplantation of EPs may be superior to mature ECs for therapeutic vascularization due to enhanced vessel formation and enhanced anastomoses with the host circulation.

In summary, Sahara et al. [4] established a conceptual framework to study stem cell differentiation through endothelial progenitors to endothelial cells. Using this framework, they developed a remarkably efficient and rapid differentiation protocol with yield (2.5 EPs for each input stem cell on day 6) and purity $(\sim 50 \%$ EPs on day 6$)$ that far exceeds previously reported protocols. In the process, the authors uncovered a new role for Notch signaling in maintaining EPs, and furthermore suggest that EPs may be more effective than ECs for therapeutic vascularization. These important advances set the stage for future work that will both enhance our understanding of angiogenesis and move cell therapy-based revascularization closer to reality. Before clinical use is contemplated, systematic efforts will be required to establish the safety of these pluripotent stem cell-derived EPs and ECs and to exclude potential adverse effects such as teratoma formation by incompletely differentiated cells. We need to further enhance the efficiency and yield of this protocol and extend it to iPSCs. The functional capacity of stem cell-derived ECs to form mature vessels with the properties of the normal circulatory system needs to be further established. Effective vascular regeneration will require understanding the signals that guide EPs to differentiate into artery, vein, capillary and lymphatic EC sublineages, and to acquire tissuespecific EC properties and expression profiles. While many manipulations have been shown to enhance microvascular density, we have limited ability to form large vessels such as coronary arteries, and perhaps interdisciplinary approaches using stem cell-derived EPs and bioengineered scaffolds will be able to fill this void. Finally, it will be interesting to determine whether combined delivery of EPs and stem cell-derived organ-specific cells such as cardiomyocytes will enable more robust organ regeneration than delivery of either cell type in isolation.

\section{Bing Zhang ${ }^{1}$, William T Pu ${ }^{1,2}$}

${ }^{1}$ Department of Cardiology, Boston Children's Hospital, Boston, MA 02115, USA; ${ }^{2}$ Harvard Stem Cell Institute, Harvard University, Cambridge, MA 02138, USA

Correspondence: Bing Zhanga, William T Pu ${ }^{\mathrm{b}}$

${ }^{a}$ E-mail: bingzhang@enders.tch.harvard.edu

bE-mail: wpu@pulab.org

\section{References}

1 Wang ZZ, Au P, Chen T, et al. Nat Biotechnol 2007; 25:317-318.

2 Yamashita J, Itoh $\mathrm{H}$, Hirashima M, et al. $\mathrm{Na}$ ture 2000; 408:92-96.

3 Levenberg S, Golub JS, Amit M, et al. Proc Natl Acad Sci USA 2002; 99:4391-4396.

4 Sahara M, Hansson EM, Wernet O, et al. Cell Res 2014; 24:820-841.

5 Samuel R, Daheron L, Liao S, et al. Proc Natl Acad Sci USA 2013; 110:12774-12779.

6 Tung JJ, Tattersall IW, Kitajewski J. Cold Spring Harb Perspect Med 2012; 2:a006601. 